\title{
Fiducial-free CyberKnife stereotactic body radiation therapy for single vertebral body metastases: acceptable local control and normal tissue tolerance with five-fraction approach
}

\author{
Beant Gill ${ }^{1}$, Eric Oermann ${ }^{1}$, Andrew Ju${ }^{1}$, Simeng Suy ${ }^{1}$, Xia Yu ${ }^{1}$, Jennifer Rabin ${ }^{1}$, Christopher Kalhorn ${ }^{2}$, \\ Mani N. Nair ${ }^{2}$, Jean-Marc Voyadzis ${ }^{2}$, Keith Unger ${ }^{1}$, Sean P. Collins ${ }^{1},{ }^{1}$ K. W. Harter ${ }^{1}$ and Brian T. Collins ${ }^{1}{ }^{*}$ \\ ${ }^{1}$ Department of Radiation Medicine, Georgetown University Hospital, Washington, DC, USA \\ ${ }^{2}$ Department of Neurosurgery, Georgetown University Hospital, Washington, DC, USA
}

\section{Edited by:}

Charles Kunos, Case Western Reserve University and University Hospitals of Cleveland, USA

Reviewed by:

Charles Kunos, Case Western

Reserve University and University Hospitals of Cleveland, USA

Aaron Howard Wolfson, University of

Miami Miller School of Medicine, USA

\section{${ }^{*}$ Correspondence:}

Brian T. Collins, Department of

Radiation Medicine, Georgetown

University Hospital, LL Bles, 3800

Reservoir Road, N.W., Washington,

DC 20007, USA. e-mail:

collinsb@gunet.georgetown.edu
This retrospective analysis examines the local control and toxicity of five-fraction fiducialfree CyberKnife stereotactic body radiation therapy (SBRT) for single vertebral body (VB) metastases. All patients had favorable performance status (ECOG 0-1), oligometastatic disease, and no prior spine irradiation. A prescribed dose of 30-35 Gy was delivered in five fractions to the planning target volume (PTV) using the CyberKnife with X-sight spine tracking. Suggested maximum spinal cord and esophagus point doses were 30 and $40 \mathrm{~Gy}$, respectively. A median $30 \mathrm{~Gy}$ (IQR, 30-35 Gy) dose was delivered to a median prescription isodose line of $70 \%(I Q R, 65-77 \%)$ to 20 patients. At 34 months median follow-up (IQR, 25-40 months) for surviving patients, the 1- and 2-year Kaplan-Meier local control estimates were 80 and $73 \%$, respectively. Two of the five local failures were infield in patients who had received irradiation to the gross tumor volume and three were paravertebral failures just outside the PTV in patients with prior corpectomy. No local failures occurred in patients who completed VB radiation alone. The 1- and 2-year Kaplan-Meier overall survival estimates were 80 and $57 \%$, respectively. Most deaths were attributed to metastatic disease; one death was attributed to local recurrence. The mean maximum point doses were $26.4 \mathrm{~Gy}$ (SD, 5.1 Gy) to the spinal cord and $29.1 \mathrm{~Gy}$ (SD, 8.9 Gy) to the esophagus. Patients receiving maximum esophagus point doses greater than $35 \mathrm{~Gy}$ experienced acute dysphagia (Grade I/II). No spinal cord toxicity was documented. Five-fraction fiducial-free CyberKnife SBRT is an acceptable treatment option for newly diagnosed VB metastases with promising local control rates and minimal toxicity despite the close proximity of such tumors to the spinal cord and esophagus. A prospective study aimed at further enhancing local control by targeting the intact $\mathrm{VB}$ and escalating the total dose is planned.

Keywords: vertebral body, metastases, stereotactic body radiation therapy, CyberKnife, spine

\section{INTRODUCTION}

Annually, 5-10\% of patients with systemic cancer will develop symptomatic spine metastases (Ries et al., 2008). Patients with common malignancies such as breast, prostate, and lung cancer develop spine metastases at higher rates. The management of vertebral body $(\mathrm{VB})$ metastases varies depending on an individual's prognosis and the involved spine's structural integrity. Patients carrying a poor prognosis without evidence of bony retropulsion into the anterior epidural space or spinal instability have traditionally received conventional radiation therapy alone with moderate success (Faul and Flickinger, 1995). Despite providing excellent early palliation of symptoms, conventional radiation has resulted in poor local control rates with one major survey placing the crude, overall local control rate at $77 \%$, and another large Phase III study estimating a median time to recurrence of 5 months (Maranzano and Latini, 1995; Moulding et al., 2010).
Patients remain at significant risk of developing debilitating spine progression following conventional irradiation (Ryu et al., 2007; Gibbs et al., 2009).

In an effort to enhance local control and minimize radiation toxicity researchers in the past decade have explored various means of improving spine irradiation. This research has resulted in a new treatment paradigm, stereotactic body radiation therapy (SBRT). With improved dose distributions and accuracy, SBRT has facilitated the safe painless delivery of high doses of radiation quickly to localized spine tumors. Multiple studies have confirmed the efficacy of SBRT with early symptomatic improvement seen in $86-96 \%$ of treated patients following delivery of one to five fractions (Gerszten et al., 2004, 2007; Ryu et al., 2004, 2008; Degen et al., 2005; Yamada et al., 2005, 2008; Chang et al., 2007; Gibbs et al., 2007; Gagnon et al., 2009). Despite growing evidence supporting SBRT in poor prognosis, previously irradiated spine 
metastases patients, few studies have established local control rates for newly diagnosed relatively good prognosis patients with single VB metastases.

The CyberKnife ${ }^{\circledR}$ System (Accuray Incorporated, Sunnyvale, CA, USA) has been successfully employed at Georgetown University Hospital since early 2002 to treat spine tumors with fiducial tracking. Institutional reports have documented this technology's ability to reliably palliate tumor induced spine pain with potentially safer fractionated radiation doses (Degen et al., 2005; Gagnon et al., 2009). The accuracy and flexibility of the fiducial-free system, $\mathrm{X}$-sight spine tracking, prompted us in late 2005 to evaluate a fivefraction CyberKnife SBRT treatment for good prognosis patients with single VB metastases that were not previously irradiated (Muacevic et al., 2006; Ho et al., 2007). The system's unique configuration facilitates the accurate delivery of fractionated high dose radiation, despite the close proximity of such tumors to radiation sensitive structures such as the spinal cord and esophagus (Kilby et al., 2010). We report outcomes for 20 consecutively treated, eligible patients with newly diagnosed single VB metastases treated using this novel treatment approach.

\section{MATERIALS AND METHODS ELIGIBILITY}

This retrospective analysis of an established departmental treatment approach was approved by the MedStar Health Research Institute - Georgetown University Oncology Institutional Review Board. Consecutive patients with good prognosis single VB metastases treated with five-fraction fiducial-free CyberKnife SBRT between September 2005 and September 2010 were analyzed. Patients were considered to have a good prognosis if they had a favorable performance status (ECOG 0-1) and oligometastatic disease. A tumor was considered a single VB metastasis if it primarily involved a single VB. Patients with previously irradiated spine or uncorrected spinal cord compression were excluded from analysis.

\section{TREATMENT PLANNING AND DELIVERY}

A fine-cut $(1.25 \mathrm{~mm})$ contrast enhanced treatment planning CT scan was obtained in the supine treatment position for each patient. Oral contrast was routinely used to delineate the esophagus. CT myelogram was used to define the spinal cord when clinically indicated. The planning target volume (PTV), either the gross tumor volume (GTV), or the involved VB, was contoured at the discretion of the treating physician. A treatment plan was generated using the MultiPlan 5.2.1 non-isocentric, inverse-planning algorithm with tissue density heterogeneity corrections for lung based on an effective depth correction. The radiation dose was divided into five equal fractions of 6-7 Gy, prescribed to an isodose line that covered at least $80 \%$ of the PTV. Guidelines for dose limits to critical structures are provided in Table 1. Patients were treated in the supine position as previously described using fiducial-free CyberKnife spine tracking (Muacevic et al., 2006; Ho et al., 2007).

\section{FOLLOW-UP}

Patients were followed with physical examination plus MRI and/or PET/CT imaging at 3- to 6-month intervals per routine
Table 1 | Institutional maximum point dose limit guidelines for adjacent critical structures.

\begin{tabular}{ll}
\hline Adjacent critical structure & $\begin{array}{l}\text { Maximum point dose limit } \\
\text { (Gy; total for five fractions) }\end{array}$ \\
\hline Spinal cord & 30 \\
Nerve roots & 35 \\
Esophagus & 40 \\
Bowel & 40 \\
\hline
\end{tabular}

institutional practice. Local tumor recurrence was defined as progression of the treated tumor per selected imaging. Biopsies were obtained when clinically indicated. Toxicities were scored according to the National Cancer Institute Common Terminology Criteria for Adverse Events, Version 3.0 (National Institutes of Health, 2006).

\section{STATISTICAL ANALYSIS}

Statistical analysis was performed with the SPSS 17 (IBM Corporation, Armonk, NY, USA) statistical software package. The follow-up duration was defined as the time from the date of treatment completion to the last date of follow-up or the date of death. Actuarial local control and overall survival were calculated using the Kaplan-Meier method. For the sake of robustness, all reported ranges are interquartile $\left(\mathrm{IQR}, \mathrm{Q}_{1}-\mathrm{Q}_{3}\right)$.

\section{RESULTS \\ PATIENT CHARACTERISTICS}

Twenty consecutive patients, 6 men and 14 women, were treated over a 60-month period and followed for a minimum of 12 months or until death (Table 2). Primary cancers included sarcoma $(n=7)$, breast cancer $(n=5)$, non-small cell lung cancer $(n=2)$, prostate cancer $(n=1)$, clear cell renal cancer $(n=1)$, papillary thyroid cancer $(n=1)$, melanoma $(n=1)$, plasmacytoma $(n=1)$, and acinic cell salivary gland cancer $(n=1)$. All patients were chemotherapy candidates; $30 \%$ required corpectomy prior to CyberKnife treatment.

\section{TREATMENT CHARACTERISTICS}

Treatment plans were composed of hundreds of pencil beams delivered using a single 10-35 mm diameter collimator (median, $20 \mathrm{~mm})$. The majority of patients $(60 \%)$ received VB irradiation; the remainder had the GTV alone targeted. The mean PTV was $121 \mathrm{cc}$ (SD, $116 \mathrm{cc}$ ). Radiation was delivered in five equal fractions of 6-7 Gy over a median of 7 days (IQR, 59 days). The median prescription isodose line was $70 \%$ (IQR, 65-77\%), the median dose was 30 Gy (IQR, 30-35 Gy) and the median PTV coverage was 95\% (IQR, 87-97\%). The resulting mean maximum point doses delivered to the spinal cord and esophagus were 26.4 Gy (SD, 5.1 Gy) and 29.1 Gy (SD, 8.9 Gy), respectively.

\section{LOCAL CONTROL AND SURVIVAL}

Local control and survival outcomes were favorable (Figure 1; Table 3). At a median follow-up of 34 months for surviving 
Table 2 | Treatment characteristics.

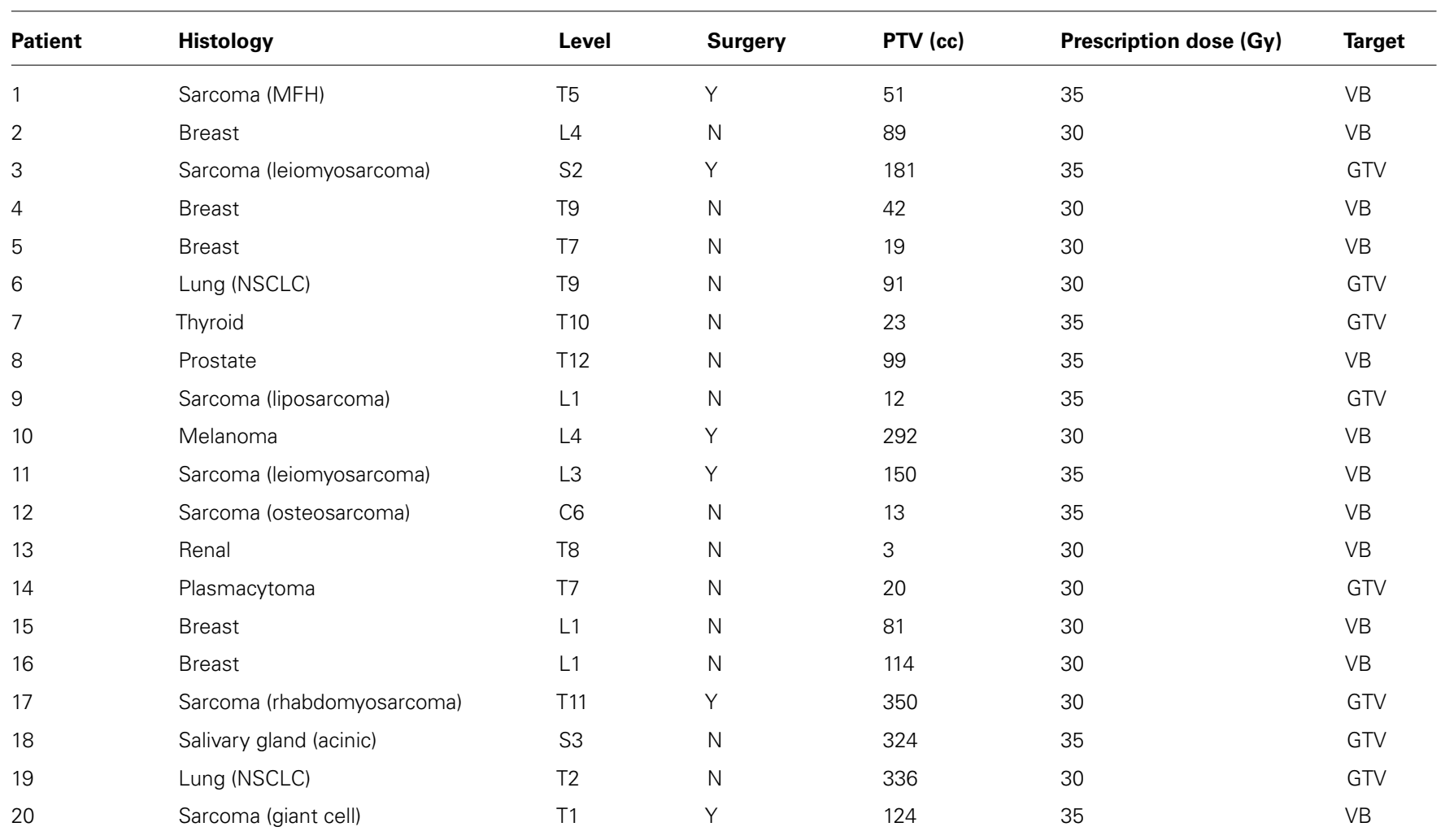

GTV, gross tumor volume; NSCLC, non-small cell lung cancer; MFH, malignant fibrous histiocytoma; VB, vertebral body.

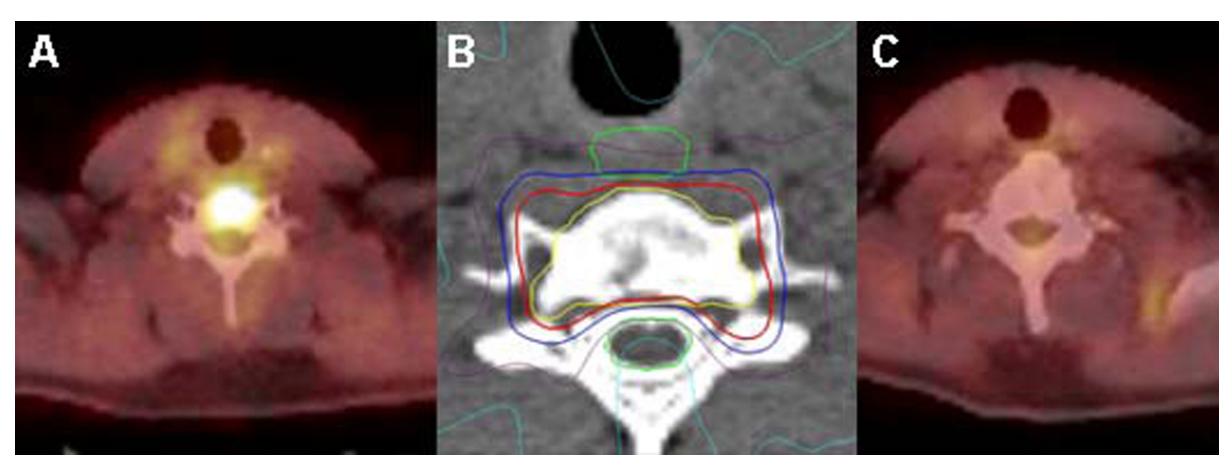

FIGURE 1 | Osteosarcoma C6 oligometastasis PET/CT scan with a tumor SUVmax of 11 (A); (B) CyberKnife treatment plan showing the vertebral body planning treatment volume in yellow and the $35 \mathrm{~Gy}$ isodose line in blue; and (C) PET/CT scan at 3 years post-treatment shows a durable complete response with a tumor SUVmax of 1.7 .

patients, the 1- and 2-year Kaplan-Meier local control estimates were 80 and $73 \%$, respectively (Figure 2). Of the five observed local failures, two were infield local failures in patients who had received irradiation to the GTV alone and three were paravertebral failures just outside the PTV in patients requiring corpectomy prior to treatment. Four of the seven treated sarcomas failed locally. No local failures were identified beyond 14 months or in patients who completed VB irradiation alone. The 1- and 2-year KaplanMeier overall survival estimates were relatively favorable at 80 and $57 \%$, respectively (Figure 3). Nine deaths were attributed to metastatic disease; one death was attributed to local recurrence (Figure 4).

\section{COMPLICATIONS}

Conservative maximum point dose guidelines for normal tissues were generally observed. Patients receiving maximum esophageal point doses greater than $35 \mathrm{~Gy}(n=4)$ experienced acute dysphagia/esophagitis (Grade I/II) lasting several weeks. No skin, musculoskeletal or neurologic toxicities were attributed to CyberKnife treatment. 
Table 3 | Clinical outcomes.

\begin{tabular}{lllll}
\hline Patient & $\begin{array}{l}\text { Local failure } \\
\text { (month) }\end{array}$ & Vital status & $\begin{array}{l}\text { Survival } \\
\text { (month) }\end{array}$ & Cause of death \\
\hline 1 & 7 & Dead & 36 & Metastases \\
2 & - & Dead & 27 & Metastases \\
3 & - & Dead & 20 & Metastases \\
4 & - & Dead & 18 & Metastases \\
5 & - & Dead & 12 & Metastases \\
6 & 5 & Dead & 12 & Metastases \\
7 & - & Alive & 63 & - \\
8 & - & Alive & 50 & - \\
9 & 14 & Dead & 24 & Local failure \\
10 & - & Alive & 40 & - \\
11 & 12 & Alive & 40 & - \\
12 & - & Alive & 39 & - \\
13 & - & Dead & 14 & Metastases \\
14 & - & Alive & 28 & - \\
15 & - & Alive & 25 & - \\
16 & - & Alive & 25 & - \\
17 & 6 & Dead & 10 & Metastases \\
18 & - & Alive & 14 & - \\
19 & - & Dead & 6 & Metastases \\
20 & - & Alive & 12 & - \\
\hline & & & & \\
\hline
\end{tabular}

\section{DISCUSSION}

The CyberKnife's image guidance system uniquely facilitates both extreme accuracy ( $<1 \mathrm{~mm}$ ) and fractionation (Kilby et al., 2010). In our opinion, both capabilities are necessary to optimally treat tumors in close proximity to the spinal cord. Georgetown University Hospital researchers have documented the CyberKnife's ability to reliably palliate tumor induced spine pain with potentially safer fractionated radiation doses using fiducial tracking (Degen et al., 2005; Gagnon et al., 2009). However, the required fiducial placement uniformly necessitated a delay in treatment and was a potentially hazardous invasive procedure that concerned potential users. In 2005 the CyberKnife image guidance system was reconfigured to track the spine itself (Muacevic et al., 2006; Ho et al., 2007). This development led to the broad adoption of CyberKnife spine SBRT.

With the introduction of fiducial-free CyberKnife spine technology to Georgetown University Hospital in 2005, we instituted a five-fraction treatment approach for newly diagnosed single VB oligometastases. This fractionation schedule was selected to optimize local control and normal tissue radiation tolerance in this group of patients with expected favorable survival. Twenty patients were treated in 60 months and followed for a minimum of 1 year. At a median follow-up of 34 months for surviving patients, the 1and 2-year Kaplan-Meier local control estimates are 80 and 73\%, respectively. This is somewhat lower than the typical $90 \%$ local control rates reported by prior institutional series (Gerszten et al., 2009). Although this small decrement in local control is likely
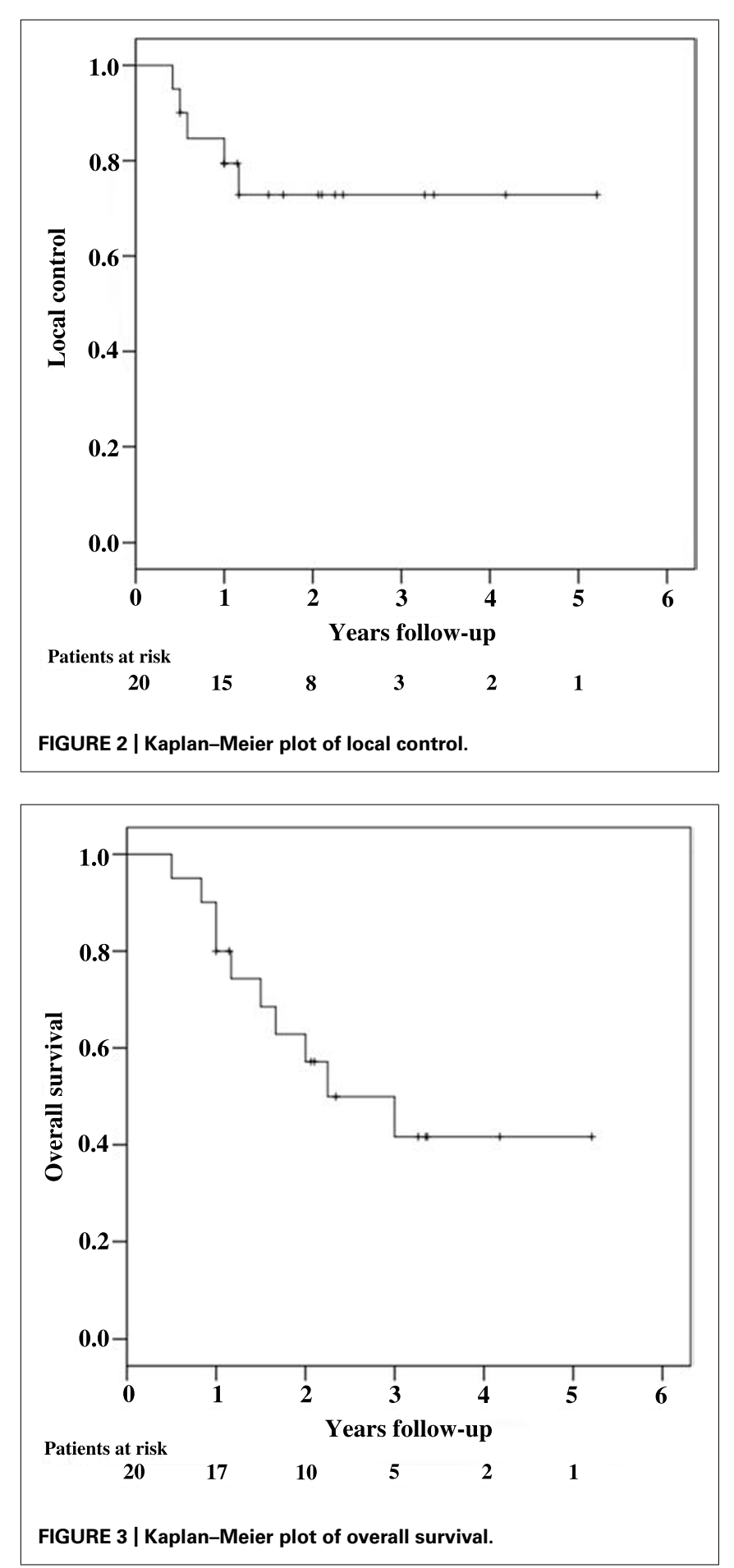

the result of the improved survival seen in this cohort, several minor adjustments to our approach have been made in an effort to improve this outcome.

Prior to proceeding with our five-fraction fiducial-free CyberKnife approach for single VB metastases there was no standard PTV for spine SBRT. Therefore, we left it to the treating physician to determine whether they would target the GTV or the VB itself. The majority of physicians $(60 \%)$ chose to treat the VB 


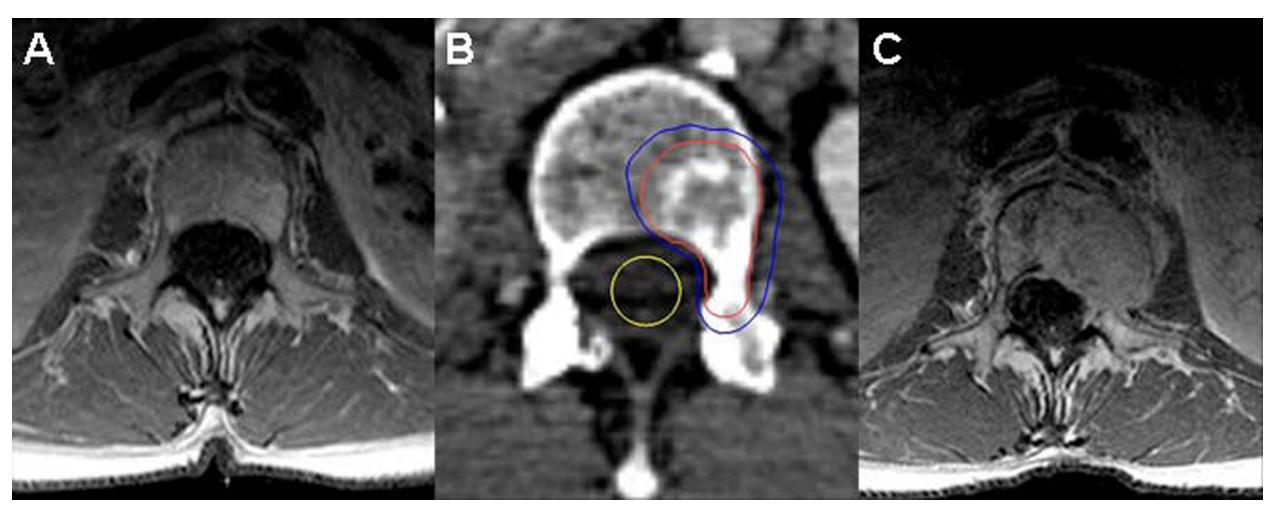

FIGURE 4 | (A) Solitary L1 liposarcoma metastasis; (B) CyberKnife treatment plan dose distribution with gross tumor volume shown in red and the 35 Gy isodose line in blue; and (C) MRI at 14 months post-treatment with local recurrence.

in order to address microscopic disease spread within the VB itself. Two local failures in this study were attributed to irradiation of only the GTV; future patients will receive VB irradiation only.

The remaining three local failures were paravertebral failures just outside the PTV in patients with advanced spine metastases requiring corpectomy. The local failure rate for postoperative patients in this study was $50 \%$. Therefore, we have decided to exclude such patients from this approach in the future; it is our opinion that good prognosis patients with likely intraoperative microscopic disease spread would be better treated with a combination of conventional radiation and CyberKnife as a boost.

Finally, it is likely that good prognosis patients with single VB metastases would benefit from dose escalation (Yamada et al., 2008). The five local failures observed in this study were seen in patients with tumors that were generally considered radioresistant (Tables 2 and 3; Gerszten et al., 2009). Furthermore, meaningful toxicity was observed only in patients receiving maximum

\section{REFERENCES}

Chang, E. L., Shiu, A. S., Mendel, E., Mathews, L. A., Mahajan, A., Allen, P. K., Weinberg, J. S., Brown, B. W., Wang, X. S., Woo, S. Y., Cleeland, C., Maor, M. H., and Rhines, L. D. (2007). Phase I/II study of stereotactic body radiotherapy for spinal metastasis and its pattern of failure. J. Neurosurg. Spine 7, 151-160.

Degen, J. W., Gagnon, G. J., Voyadzis, J. M., Mcrae, D. A., Lunsden, M., Dieterich, S., Molzahn, I., and Henderson, F. C. (2005). CyberKnife stereotactic radiosurgical treatment of spinal tumors for pain control and quality of life. J. Neurosurg. Spine 2, 540-549.

Faul, C. M., and Flickinger, J. C. (1995). The use of radiation in the management of spinal metastases. J. Neurooncol. 23, 149-161.

Gagnon, G. J., Nasr, N. M., Liao, J. J., Molzahn, I., Marsh, D., Mcrae,
D., and Henderson, F. C. Sr. (2009). Treatment of spinal tumors using cyberknife fractionated stereotactic radiosurgery: pain and quality-oflife assessment after treatment in 200 patients. Neurosurgery 64, 297-306; discussion 306-307.

Gerszten, P. C., Burton, S. A., Ozhasoglu, C., and Welch, W. C. (2007). Radiosurgery for spinal metastases: clinical experience in 500 cases from a single institution. Spine (Phila $\mathrm{Pa}$ 1976) 32, 193-199.

Gerszten, P. C., Mendel, E., and Yamada, Y. (2009). Radiotherapy and radiosurgery for metastatic spine disease: what are the options, indications, and outcomes? Spine (Phila Pa 1976) 34, S78-S92.

Gerszten, P. C., Ozhasoglu, C., Burton, S. A., Vogel, W. J., Atkins, B. A., Kalnicki, S., and Welch, W. C. (2004). CyberKnife frameless stereotactic radiosurgery for spinal lesions: clinical experience in 125 cases.

esophagus point doses greater than $35 \mathrm{~Gy}$. A planned institutional dose escalation study will gradually raise the current typically dose limiting maximum spinal cord point dose from 30 to $35 \mathrm{~Gy}$ as tolerated. The current esophagus maximum point dose will be decreased to $35 \mathrm{~Gy}$ to minimize acute dysphagia.

\section{CONCLUSION}

Five-fraction fiducial-free CyberKnife SBRT is a promising treatment option for VB tumors, demonstrating encouraging local control rates with nominal toxicity despite the close proximity of such tumors to the spinal cord and esophagus. A prospective dose escalation study targeting the intact VB is planned to further enhance local control in this patient population.

\section{ACKNOWLEDGMENT}

Eric Oermann is supported by a Doris Duke Charitable Research Foundation Clinical Research Fellowship.

Neurosurgery 55, 89-98; discussion 98-99.

Gibbs, I. C., Kamnerdsupaphon, P., Ryu, M. R., Dodd, R., Kiernan, M., Chang, S. D., and Adler, J. R. Jr. (2007). Image-guided robotic radiosurgery for spinal metastases. Radiother. Oncol. 82, 185-190.

Gibbs, I. C., Patil, C., Gerszten, P. C., Adler, J. R. Jr., and Burton, S. A. (2009). Delayed radiation-induced myelopathy after spinal radiosurgery. Neurosurgery 64, A67-A72.

Ho, A. K., Fu, D., Cotrutz, C., Hancock, S. L., Chang, S. D., Gibbs, I. C., Maurer, C. R. Jr., and Adler, J. R. Jr. (2007). A study of the accuracy of cyberknife spinal radiosurgery using skeletal structure tracking. Neurosurgery 60, ONS147-ONS156; discussion ONS156.

Kilby, W., Dooley, J. R., Kuduvalli, G., Sayeh, S., and Maurer, C. R. Jr. (2010). The CyberKnife Robotic Radiosurgery System in
2010. Technol. Cancer Res. Treat. 9, 433-452.

Maranzano, E., and Latini, P. (1995). Effectiveness of radiation therapy without surgery in metastatic spinal cord compression: final results from a prospective trial. Int. J. Radiat. Oncol. Biol. Phys. 32, 959-967.

Moulding, H. D., Elder, J. B., Lis, E., Lovelock, D. M., Zhang, Z., Yamada, Y., and Bilsky, M. H. (2010). Local disease control after decompressive surgery and adjuvant highdose single-fraction radiosurgery for spine metastases. J. Neurosurg. Spine 13, 87-93.

Muacevic, A., Staehler, M., Drexler, C., Wowra, B., Reiser, M., and Tonn, J. C. (2006). Technical description, phantom accuracy, and clinical feasibility for fiducial-free frameless real-time image-guided spinal radiosurgery. J. Neurosurg. Spine 5, 303-312. 
National Institutes of Health. (2006). Common Toxicity Criteria for Adverse Events, Version 3.0. Available at: http://ctep.cancer.gov/protocolDevel opment/electronic_applications/ docs/ctcaev3.pdf

Ries, L. A. G., Melbert, D., Krapcho, M., Stinchcomb, D. G., Howlader, N., Horner, M. J., Mariotto, A., Miller, B. A., Feuer, E. J., Altekruse, S. F., Lewis, D. R., Clegg, L., Eisner, M. P., Reichman, M., and Edwards, B. K. (eds). (2008). SEER Cancer Statistics Review, 1975-2005 [Online]. Bethesda, MD: National Cancer Institute. Available at: http://seer.cancer. gov/csr/1975_2005/ (accessed January 23, 2012).

Ryu, S., Jin, J. Y., Jin, R., Rock, J., Ajlouni, M., Movsas, B., Rosenblum, M., and Kim, J. H. (2007). Partial volume tolerance of the spinal cord and complications of single-dose radiosurgery. Cancer 109, 628-636.

Ryu, S., Jin, R., Jin, J. Y., Chen, Q., Rock, J., Anderson, J., and Movsas, B. (2008). Pain control by image-guided radiosurgery for solitary spinal metastasis. J. Pain Symptom Manage. 35, 292-298.

Ryu, S., Rock, J., Rosenblum, M. and Kim, J. H. (2004). Patterns of failure after single-dose radiosurgery for spinal metastasis. J. Neurosurg. 101(Suppl. 3), 402-405.

Yamada, Y., Bilsky, M. H., Lovelock, D. M., Venkatraman, E. S., Toner, S., Johnson, J., Zatcky, J., Zelefsky, M. J., and Fuks, Z. (2008). High-dose, single-fraction imageguided intensity-modulated radiotherapy for metastatic spinal lesions. Int. J. Radiat. Oncol. Biol. Phys. 71, 484-490.
Yamada, Y., Lovelock, D. M., Yenice, K. M., Bilsky, M. H., Hunt, M. A., Zatcky, J., and Leibel, S. A. (2005). Multifractionated imageguided and stereotactic intensitymodulated radiotherapy of paraspinal tumors: a preliminary report. Int. J. Radiat. Oncol. Biol. Phys. 62, 53-61.

Conflict of Interest Statement: Brian T. Collins and Sean P. Collins have received honorarium from Accuray Inc. for speaking engagements.

Received: 07 February 2012; accepted: 05 April 2012; published online: 26 April 2012.

Citation: Gill B, Oermann E, Ju A, Suy $S, Y u$ X, Rabin J, Kalhorn $C$,
Nair MN, Voyadzis J-M, Unger $K$, Collins SP, Harter KW and Collins BT (2012) Fiducial-free CyberKnife stereotactic body radiation therapy for single vertebral body metastases: acceptable local control and normal tissue toler ance with five-fraction approach. Front. Oncol. 2:39. doi: 10.3389/fonc.2012. 00039

This article was submitted to Frontiers in Radiation Oncology, a specialty of Frontiers in Oncology.

Copyright (C) 2012 Gill, Oermann, Ju, Suy, Yu, Rabin, Kalhorn, Nair, Voyadzis, Unger, Collins, Harter and Collins. This is an open-access article distributed under the terms of the Creative Commons Attribution Non Commercial License, which permits non-commercial use, distribution, and reproduction in other forums, provided the original authors and source are credited. 